\title{
AQU Catalunya: Una herramienta para la mejora continuada del Sistema Universitario Catalán
}

\author{
"AQU Catalunya": A tool for continual improvement \\ of Catalan University System
}

\begin{abstract}
Martí Cassadesús Fa
Doctor ingeniero industrial y catedrático de Universidad de Organización de Empresas de la Universidad de Girona. Actualmente es director de la Agencia para la Calidad del Sistema Universitario de Catalunya (AQU Catalunya).

Email: direccio@aqu.cat

\section{Núria Comet Señal}

Licenciada en Ciencias Químicas y Licenciada en Documentación Responsable de Calidad Interna y Gestora de Proyectos de AQU Catalunya.

Email: direccio@aqu.cat
\end{abstract}

\section{Resumen}

Este artículo resume la estructura, funciones y actividades de AQU Catalunya, como agen cia de calidad universitaria cuya finalidad principal es la de promover y garantizar la calidad dela educación superior, de acuerdo con estándares académicos y sociales intern acion ales y europ eos de calid ad y de con formid ad con la normativa vigente, proporcion and o a los agentes de la edu cación sup erior criterios y referentes para alcanzar los máximos están dares de calidad en el cumplimien to de sus funciones, satisfaciendo el interés que la sociedad tiene por una educación superior de calidad. 
El artículo resume la actividad principal de 4 de los 6 ejes estratégicos más relevantes: Calidad institucional, Calidad del profesorado universitario, Generación del conocimiento, e Internacionalización.

\section{Palabras clave}

Agencias de calidad; calidad en la educación superior, asegu ramiento de la calidad; AQU Catalunya; universidades.

\section{Abstract}

This article summarizes the structure, functions and activities of AQU Catalunya, like agency of university quality the main purpose of which is promoting and guaranteeing the quality of higher education, in accordance with international and European academic and social stand ard s of quality and in accord ance with the valid regulations, and to provide to the agents of high er education criteria and referents to attain the highest standards of quality in the fulfilment of its functions, satisfying the interest that the society has for an education of quality.

The article summarizes the main activity of 4 of the 6 more relevant strategic priorities: Institutional quality, Quality of the university teaching staff, Generation of the knowledge, and Internationalization.

\section{Key words}

Agencies of quality; higher education quality; quality assuran ce; AQU Catalunya, un iversities. 
AQU Catalunya: Una herramienta para la mejora continuada del Sistema Univers itario Catalán

\section{Entorno: el Sistema Universitario Catalán y el aseguramiento de la calidad}

AQU Catalu nya es la agen cia de calid ad universitaria del Sistema Universitario Catalán. Este cuenta con doce universidades, siete de las cuales son públicas, cuatro privadas y una responde a un modelo mixto. Cuatro de las universidades públicas concentran sus campus en Barcelona y su área metropolitana: la Universidad de Barcelona (UB, 1837), la Universidad Autónoma de Barcelona (UAB, 1968), la Universidad Politécnica de Catalunya (UPC, 1971) y la Universidad Pompeu Fabra (UPF, 1990). Las tres públicas restantes tien en su área de influ encia en las otras tres capitales catalanas: la Universidad de Girona (UdG, 1990), la Universidad de Lleida (UdL, 1990) y, en Tarragona, la Universidad Rovira i Virgili (URV, 1990). La Universidad Oberta de Catalunya (UOC, 1995), que imparte en señanzas a distancia, es una universidad privada regida por una fundación participada mayoritariamente por el gobierno de la Generalitat de Catalunya. Completan el sistema la Universidad Ramon Llull (URL, 1991), la Universidad de VicUniversidad Central de Catalunya (UVic, 1997), la Universidad Internacional de Catalunya (UIC, 1997) y la Universidad Abad Oliba-CEU (UAO, 2003), las cuatro de titularidad privada, y tres de las cuales tienen sede en Barcelona y una en la ciudad de Vic.

En el curso 2014-2015 había 205.830 estudiantes matriculados en las universidades catalanas, el $71 \%$ en in stituciones públicas, y 17.413 profesores y profesoras. La tabla 1 muestra la dimensión del Sistema Universitario Catalán, en número de estudiantes y profesores (PDI).

Tabla 1. La dimensión del sistema universitario catalán.

\begin{tabular}{|l|r|r|}
\hline Unive rsidad & Estudiantado* & Profesorado*** $^{* *}$ \\
\hline UB & 47.270 & 4.845 \\
\hline UAB & 30.141 & 3.338 \\
\hline UPC & 25.838 & 2.604 \\
\hline UPF & 10.778 & 897 \\
\hline UdG & 11.403 & 1.169 \\
\hline UdL & 8.290 & 957 \\
\hline URV & 12.624 & 1.613 \\
\hline UOC & 34.334 & 249 \\
\hline URL & 15.297 & 950 \\
\hline UVic-UCC & 4.729 & 442 \\
\hline UIC & 3.335 & 298 \\
\hline UAO & 1.791 & 196 \\
\hline Total & $\mathbf{2 0 5 . 8 3 0}$ & $\mathbf{1 7 . 5 5 8}$ \\
\hline
\end{tabular}

Fuente: Sistema de información Winddat

* Datos del curso 2014-2015; ** Datos del curso 2013-2014 
La educación superior es una materia regulada tanto por la Constitución española como por el Estatuto de Autonomía de Catalunya. La distribución competencial de esta materia corresponde en exclusiva al Estado en lo referente al fomento y coordinación general de la investigación científica y técnica, las bases de régimen del estatu to de los funcionarios, yla regulación de las condiciones de la obtención, la expedición y la homologación de títu los acad émicos y profesionales y las normas bá sicas para el desarrollo del derech o a la edu cación y de la au tonomía universitaria. Asimismo, corresponde a la comunidad autónoma de Catalunya la competencia exclusiva, entre otros, de la programación y la coordinación del sistema universitario catalán, el marco jurídico de los títu los propios de las universidades, la financiación propia de las universid ades, el régimen retributivo del personal d ocente e investigad or con tratado por las un iversidades y el establecimien to de las condiciones de las retribucio nes adicionales del personal docen te funcionario. Por otra parte, son competencias compartidas entre el Estado y la comunidad au tónoma de Catalu nya la regulación de los requisitos para la creación y el reconocimiento de universidades, el régimen jurídico de la organización y el funcionamiento de las universidades públicas, la regulación del régimen del profesorado docente investigad or con tratad oy funcionario, yla evaluación yel aseguramien to de la calidad y la excelen cia de la en señanza universitario, así como también del personal docente e investigador.

El despliegue normativo de la enseñanza superior se ha articulado, principalmente, a través de la Ley orgánica 6/2001, de 21 de diciembre, de universid ades, modificada por la Ley orgánica 4/2007, de 12 de abril (LOMLOU), que determina las condiciones básicas de la mayoría de los aspectos de la enseñanza universitaria. Las competencias en Catalunya se desarrollan mediante la Ley 1/2003, de 19 de febrero, de universidades de Catalunya (LUC).

Así pues, de acuerdo con las disposiciones normativas citadas, la LUC define el sistema un iversitario de Catalu nya como aquel in tegrad o porlas un iversid ad es establecidas en Catalunya y detalla los objetivos y los principios in formadores; también trata de la actividad universitaria, que comp ren de la docencia y la in vestigación; d efine la comunidad universitaria como la integrada por el estudiantado, el personal docente e investigador, y el personal de administración y servicios; hace referencia al gobierno y la representación de las universidad es públicas y al régimen ju rídico de las universid ades y la ord enación de los estudios y estructuras universitarias; trata las garantías de calidad de las universidades y, finalmen te, regula determinados aspectos del régimen econ ómico y financiero de las universidades públicas.

\section{Historia e hitos}

AQU Catalunya tien e su origen en el consorcio Agencia para la Calidad del Sistema Un iversitario en Catalunya, constituido el 29 de octubre de 1996. Con la aprobación de la LUC el año 2003, el consorcio se tran sformó en la actual Agencia, que se ha consolidado con la Ley de AQU Catalunya en el 2015. 
Fue la primera agencia de calidad que se creó en el Estado español y la primera en Europa certificada con la norma ISO 9001 (2000). En el a ño 2011 la Agencia superó satisfactoriamente la au ditoría externa correspondiente al seguimiento de la certificación ISO 9001:2008. El alcance de la certificación es el proceso de evaluación de la calidad universitaria, que incluye la evaluación institucional y la evaluación del profesorado universitario. La norma ISO 9001:2008, in tern acionalmentereconocida, contien e directrices que permiten a cualqu ier usuario diseñar e implantar un sistema de gestión de la calidad a medida orientado al aseguramien to de la calidad del producto o servicio y a aumentar la satisfacción de usuarios o clientes. En el 2015 superó la norma ISO 27001:2013 en materia de seguridad de la información.

AQU Catalunya es miemb ro fundador de ENQA (2000) y, tras superar dos evaluaciones externas internacionales de acuerdo con los ESG coordinadas por la misma asociación (2007 y 2012), es miembro de pleno derecho. La Agencia también forma parte del E uropean Quality Assurance Register for Higher Edu cation, EQAR (2008), Ia International Network for Quality Assurance Agencies in Higher Education, INQAAHE (1998), de la Red Española de Agencias de Calidad Universitarias, REACU (2006) y del Eu rop ean Consortium for Accreditation, ECA (2009).

Desde el año 2013 asume el secretariado de la INQAAHE, que está formada por casi 300 instituciones vinculadas a la educación superior de todo el mundo.

Ahora bien, definitivamente la Agencia para la Calidad del Sistema Universitario de Catalunya toma una gran relevan cia cuando en el 2015, la Ley 15/2015 de 21 de julio la regula específicamente. En esta Ley se define la finalidad de la Agencia como la de promover y garantizar la calidad de la educación superior, de acuerdo con estándares académi cos y sociales internacionales y eu ropeos de calidad y de conformidad con la normativa vigente, y proporcionar a los agentes de la educación superior criterios y referentes para alcanzar los máximos están dares de calidad en el cumplimiento de sus funciones, satisfacien do el in terés que la socied ad tien e por una edu cación su perior de calidad.

Sus objetivos concretos, según la misma ley, son:

a) Proporcionarel aseguramiento externo de la calidad de la docencia, la investigación y la innovación del sistema universitario de Catalunya y de los centros de educación superior.

b) Facilitar la garantía y mejora del mod elo universitario propio de Catalu nya y su proyección internacional

c) Dar apoyo al desarrollo de un sistema propio de enseñanzas superiores, en el ámbito de competencias de la Generalitat, con el despliegue de un marco de calificaciones que incluya programas internacionales.

d) Convertirse en una agen cia de referencia internacional en la acreditación y el aseguramien to de la calid ad, que ofrezca servicios a universidades y a otras entidades del Estad o español, eu ropeas y de todo el mundo, con atención preferente a las universidades catalanas. 
e) Desarrollarla metodología, las herramientas y las propuestas, en el ámbito de la calidad, que contribuyan a situar el sistema universitario y los centros de educación superior de Catalun ya en una posición puntera en el ámbito internacional.

f) Introducir un sistema de evaluación que identifique las mejores prácticas, que promueva cambios y que incorpore valor añadido a la evaluación.

g) Ofrecer apoyo cualificado a los agentes que intervienen en lo s procesos de introducción de innovaciones y evaluación de nuevas políticas universitarias.

Le corresponden las funciones siguientes:

a) La evaluación de la docen cia, la in vestigación, la transferencia de conocimiento y la gestión del sistema universitario.

b) La evaluación, certificación, au ditoría yacreditación de la calidad de las universidades, de sus cen tros de investigación e innovación y de los cen tros de educación superior.

c) La evaluación, la certificación, el seguimiento y la acreditación, de acuerdo con están dares acad émicos y sociales in tern acion ales, de las en señanzas conducentes a la obtención de títulos oficiales y propios que imparten las universidad esy otros centros de educación superior.

d) El fomento de la evaluación y la comparación de criterios de calidad, de acuerdo con estándares internacionales, especialmente con respecto a la docencia, la investigación y la transferencia del conocimiento.

e) La emisión de las acreditaciones de investigación y de investigación avanzada, de acuerdo con la Ley de universidades de Catalunya.

f) La emisión de los informes pertinentes para la contratación de profesores lectores de acuerdo con la Ley de universidades de Catalunya.

g) La evaluación de la actividad del personal docentee investigad or de las universidades privadas y de los centros adscritos a las universidades.

h) La evaluación de la actividad que cumple el personal investigad or de las universidad es y la valoración de los méritos in dividuales del personal d ocentee investigador, funcionario y contratado, de acuerdo con la Ley de universidades de Catalunya.

i) La observación, el análisis y el debate sobre las ten den cias y el desarrollo en el ámbito de la educación superior, y también la orientación experta y el asesoramiento para contribuir a promover la excelencia de los servicios prestados por las universidades y los centros de educación superior. 
j) La elaboración y la comunicación de información sobre los déficits de calidad detectad os en las un iversidades, sus cen tros de investigación e in novación y los centros de educación superior, con el objetivo de mejorar la calidad.

k) La elaboración de información estadística y de indicadores sobre la educación superior y la investigación de las universidades con el fin de facilitar el análisis de su situación y evolución.

I) El impulso dela generación de conocimiento y la provisión de información pública que demuestre el estad o de la calidad y de los procesos de evaluación de la calidad de las universidades y de los centros de educación superior y de los servicios que ofrecen, para contribuir a la rendición de cuentas a la sociedad.

m) El fomento y el impulso dela investigación y la in novación sobre los modelos y los procesos de evaluación, certificación y acreditación de la calidad.

n) La representación internacional del sistema de garantía externa de calidad correspond iente al sistema universitario de Catalunya, la participación en redes u organismos $\mathrm{y}$ en instituciones $u$ organizaciones competentes en materia de aseguramiento dela calidad, y la participación en el desarrollo del Espacio Europeo de Educación Superior, todo ello de conformidad con la normativa vigente.

o) El fomento de evaluaciones temáticas y de procesos de garantía interna de calidad de las universidades y los centros de ed u cación superiory el desarrollo de procesos de evaluación y garantía externa de la calidad.

p) Los cometidos que, en el ámbito de sus funciones, le sean encargados por el departamen to competen te en materia de universidades, por las universidades, por los centros de educación superior, por los centros de investigación e innovación vinculados, por otros departamentos de la Ad min istración de la Generalitat, por institucion es o cen tros de educación superior eu ropeos y por otrasentidades que cumplen una actividad similar en las universidades catalanas.

q) Aquellas otras funciones que le atribuyen las leyes que hacen referencia a la Agencia, sus Estatu tos y otras normas vigentes.

\section{Estructura y funcionamiento de AQU Catalunya}

AQU Catalunya está constituida por órganos de gobierno (Presidencia, Dirección y Consejo de Gobierno)y órganos de evaluación (Comisión de Evaluación Institucional y de Programas, Comisión de Evaluación de la Investigación), cuya composición determina la Ley 15/2015. Los órgan os de evaluación se estructuran en comisiones específicas, que son las responsab les finales de las evaluaciones, y actúan con in dependencia técnica. Los expertos que forman parte de estas comisiones se seleccionan de acuerdo 
con el procedimiento establecido porla Agencia y son nombrados por los presidentes de los órganos de evaluación.

El presidente o presidenta de AQU Catalunya, que también lo es del Consejo de Dirección y de su Comisión Permanente, es nombrado por el gobierno de la Generalitat a propuesta de la persona titular del departamen to competente en materia de universidades, entre personalidades de reconocido prestigio en el ámbito universitario, por un periodo de cuatro años renovable. Entre sus funciones destacan:

- Velar por la consecución de los objetivos fijados.

- Ejercer la representación institucional de AQU Catalunya.

- Informar al Govern y al Parlament de Catalunya sobre la actividad de la Agencia.

El Consejo de Gobierno, que es el órgano superior de gobierno de AQU Catalunya y está constituido por el presidente o presidenta de la Agencia; los rectores y/o las rectoras de las universidades; los presidentes y/o las presidentas de los con sejos sociales de las universidades públicas; tres personas destacadas de la comunidad académica; dos estudiantes; dos personas del departamento competente en materia de universidades; el presidentey/o la presidenta de las Comisiones de Evaluación de la Investigación y el directoro directora de AQU Catalunya. Correspond en al Consejo de Gobierno, entre otras, las funciones siguientes:

- Aprobar el programa de actividades de la Agencia.

- Establecer las políticas y los objetivos estratégicos.

- Aprobar el balance, la cuenta de resultados y la memoria económica.

El Consejo de Gobierno puede actuar en pleno o en Comisión Permanente.

El director o directora de la Agencia, que asume la dirección de la Agencia y la representación plena del Consejo de Gobierno en relación con la ejecución de los acuerdos que ad op te este órgano. El directoro directora es designad o por la persona titular del departamen to competen te en materia de universidades, escuch ado el Consejo de Gobierno, a propuesta del presidente o presidenta de la Agencia, por un period o de cuatro años, prorrogable una vez. Sus funciones son las siguientes:

- Dirigir, organizar, gestionar in speccionarlos servicios de la Agencia, de acuerdo con las directrices del Consejo de Dirección.

- Ejecutary hacer cumplir los acuerd os del Consejo de Dirección y de su Comisión Permanente.

- Ostentar la representación legal de AQU Catalunya. 
Los órganos de evaluación de la Agencia son los siguientes:

La Comisión de Evaluación Institucional y de Programas (CAIP) vela porla correcta aplicación del conjunto de instrumentos y procesos de aseguramiento de la calid ad que a nivel in stitu cional realiza AQU Catalunya. Los trabajos de la CAIP se pued en agrupar en dos ejes principales:

- La calidad de las enseñanzas (titulaciones).

- La calidad de las instituciones y de los servicios.

La CAIP se estructura en seis comisiones específicas, cinco por ramas de conocimiento que son las en cargad as de evaluar los procesos de verificación, seguimiento, modificación y acreditación de las universidades catalanas, y una para valorar los sistemas de gestión in ternos de la Calidad de las Universidad es. Los presidentes y/o las presidentas de las comision es específicas son al mismo tiempo miembros de la CAIP. Esta comisión está formada por personas académicas y expertas internacionales.

La Comisión de Evaluación de la Investigación (CAR) tiene las competencias siguientes: la emisión de las acreditaciones de investigación y de investigación avanzada; la evaluación de la actividad desarrollada por los investigadores, y la valoración de los méritos in dividuales de investigación del personal docente e investigad or, funcionario y contratado; y la evaluación de la actividad investigadora del personal docente e investigador de las universidades privadas. Esta comisión está formada por personas acad émi cas de prestigio recon ocid o y desarrolla su actividad mediante comisiones específicas por ámbitos del conocimiento.

Además de los órganos de gobierno y de evaluación, y con el objetivo de coordinar las políticas y reforzar las actividades que la Agencia realiza, la dirección convoca a reuniones periódicas órganos consultivos o asesores tales como los presidentes y las presidentas y los secretarios y las secretarias de los consejos sociales de las universidades catalanas; el Comité de vicerrectores y vicerrectoras responsables de la calidad; el Comité de Unidades Técnicas de Calidad las universidades; y, desde el 2010 también el Comité d'Estudiantes. Este último trabaja para ampliar y sistematizar la participación del estudiantado en las actividades de evaluación de AQU Catalunya, asesora a la Agencia en aquellos proyectos que tienen un impacto directo sobre los estudiantes y participa en la elaboración de estudios de interés para el estudiantado. Para llevar a cabo las actividades en comendadas por la LUC y para asegurar que está en camino de cumplir con sus propósitos estratégicos, la Agencia cuenta con una estructura organizativa formada porpersonal propio que se distribuye en las áreas y las unidades siguientes:

Área de Evaluación de la Calidad. Tiene el encargo de gestionar y coordinar todas las activid ades atribu id as a la Comisión de Evaluación Institu cional y de Programas y a sus comision es específicas. Estas actividad es están relacionadas con la evaluación, la acreditación y la certificación en el ámbito de las titulaciones, las actividades, los centros, y 
los sistemas y los procesos de garantía de la calidad, y también la realización y la coordinación de estudios relacionados con estas funciones.

Área de Profesorado e Investigación. Tiene el encargo de gestionar y coordinar todas las actividad es atribuidas a la Comisión de Evaluación de la Investigación y las respect ivas comisiones que se derivan de la misma. Estas actividades están relacionadas con la emisión de informes para que las universidades públicas lleven a cabo la contratación en las categorías de profesorado lector, con la emisión de acreditacion es de investigación y de investigación avanzada, acreditación de manuales docentes y también la realización y la coordinación de estudios relacionados con estas funciones.

Unidad de Planificación y Comunicación. Tiene el encargo de gestionar y coordinar tod as las actividad es relacionadas con la comunicación, la imagen corporativa, la organización de talleres y jornadas, y el plan anual actividades.

Unidad de Administración. Tien e el encargo de gestionary coordinar el área económica y fin anciera, la gestión de los recurso s humanos, la recepción y el registro, las compras, y el mantenimiento de los espacios de AQU Catalunya.

Unidad de Informática. Tiene el encargo de gestionar y coordinar las actividad es relacionadas con las tecnologías de la información que engloban tanto las aplicaciones informáticas como las infraestructuras tecnológicas.

Apoyo a Dirección. Tiene el encargo de asesorar a Dirección en el ámbito jurídico, de calidad interna y coordinación de proyectos e incorpora, también la secretaría de dirección.

La Dirección de la Agencia también obtiene el apoyo del secretario o secretaria del Consejo de Gobierno de la Agencia y de los coordinadores y asesores.

El secretario o secretaria de la Agencia. Es nombrado por el Consejo de Gobierno a propuesta del presiden te o presidenta de la Agencia y asiste a las reuniones del Consejo de Gobierno y de la Comisión Permanente con voz pero sin voto. Sus funciones son las propias de los secretarios o secretarias de los órganos colegiados.

Coordinadores y asesores. Algunas de las actividades de la Agencia se refuerzan con personas que ocupan cargos de coordinador y asesores. Actualmente, AQU Catalunya cuenta con el apoyo de un coordinador de in novación y desarrollo metod ológico y con un asesor en materia de evaluación de programas y de evaluación institucional.

\section{Ejes de actuación}

El campo de actuación y visión de futuro de la agencia se desarrollan en el Plan estratégico de AQU Catalunya 2015-2018 (AQU, 2015), elaborado con la colaboración de las universidades y el Gobierno de la Generalitat de Catalunya, y ap robado por el Consejo de Gobierno de la Agencia. Este Plan tiene como finalidad mejorar la adecuación del 
funcionamiento de la Agencia a los objetivos definidos por la LUC y a los estándares y recomendaciones europeos.

El Plan estratégico se concreta anualmente con la Planificación de actividades de AQU Catalunya, que aprueba el Consejo de Gobierno, y ésta, a su vez, se despliega en proyectos y actividades, de los cuales se hace un seguimiento, a través del cumplimiento del calendario, indicadores, coste económico y horas destinadas que se recogen a través de diferentes aplicaciones y sistemas de gestión.

Este plan se estru ctu ra según los siguientes seis ejes (2015-2018): Calidad institu cional, Calidad del profesorado, Generación de conocimiento, Internacionalización, Dirección estratégica y comu nicación y Organización interna. En los siguientes apartados, se incluye una breve descripción de las actuaciones principales en los ejes más relevantes.

\subsection{Calidad institucional}

Con la aprobación del Real decreto 861/2010, de 2 de julio, que modifica el Real decreto $1393 / 2007$, de 29 de octubre, AQU Catalunya recuperó la potestad para emitir informes de evaluación preceptivos en el proceso de verificación de las titulaciones oficiales catalanas. La Agencia ap rovechó este impase temporal de dos años para aten der a los requerimien tos eu ro peos y desarrollary poner en práctica la metod ología de evaluación que se está aplicando actualmente en los procesos de verificación, modificación, seguimiento y acreditación de las titulaciones oficiales a través del impulso de tres programas: AUDIT, evaluación de centros adscritos y programa experimental de seguimiento de titulaciones.

Para dar apoyo a las universidades y a los centros universitarios en el diseño de sistemas de garantía in terna de la calidad de sus programas de formación, AQU Catalunya, de forma conjunta con ANECA (Agen cia Nacional de Evaluación de la Calidad y Acreditación) y ACSUG (Axencia para a Calidade do Sistema Universitario de Galicia), desarroIlaron el programa institucional, de carácter voluntario, AUDIT (2007-actualidad). Hay que tener en cuenta que una de las dimensiones que se requiere en el proceso de verificación de una nueva enseñanza es que este disponga de un Sistema de Garantía Interna de Calidad (SGIC). AQU Catalunya integra AUDIT en el Marco para la verificación, seguimiento, modificación y acreditación (VSMA), ya que eso implica que los centros velan por la calidad de sus programas de formación a través del cumplimiento de un SGIC implantado.

Con el programa de evaluación de Centros adscritos (2008-2010), y para garantizar la calidad de las propuestas de titulaciones adaptadas al EEES, la Agencia evaluó todos los centros adscritos de Catalunya (38). Este programa ha servido para fortalecer la Agencia en los p rocesos de evaluación institu cional, en los cuales se fundamen tará el proceso de las visitas externas a los centros universitarios previstas dentro del Marco VSMA como un elemento esencial del proceso de acreditación.

El programa Experimental de seguimiento (2009-2010) tenía por objetivos contribuiral diseño del procedimien to de seguimiento de las titulaciones universitarias y definirla estructura y los contenidos de los informes que anualmente tien en que elaborar las 
universidades. Participaron en el mismo todas las universidades catalanas con un máximo de dos titulaciones, una de grado y una de máster.

Los resultados de este programa se han integrado en el Marco para la verificación, el seguimiento, la modificación y la acreditación de las titulaciones (2010-actualidad) (AQU, 2010). El Marco VSMA, acordad o con los respon sables de las universidades catalanas, tiene los propósitos siguientes:

- Garantizar una evaluación continuada d el funcionamiento de las enseñanzas.

- Promover la cultura de la calidad y del rendimiento de cuentas.

- Dar apoyo a los responsables universitarios en la construcción de la visión estratégica de las enseñanzas.

- Reforzar la transparencia, el liderazgo yel reconocimien to social de la universidad.

El Marco VSMA es el principal programa de evaluación de la Agencia y el eje vertebrador de la garantía de calid ad externa de los programas de formación de las un iversid ades catalanas. Combina la evaluación de la calidad de los programas de formación con la evaluación institu cionaly da una visión estructurada e in tegrada de los cuatro procesos de aseguramiento de la calidad que se suceden cíclicamente (verificación, seguimiento, modificación y acreditación):

- Acreditación ex ante (verificación). Antes de poner en funcionamiento una nueva titulación, las universidades tien en que someter la propuesta de plan de estudios a la evaluación previa de una agencia española que sea miembro de ENQA y de EQAR. AQU Catalun ya tiene las atribuciones legales y la competencia técnica para emitir los informes previos de evaluación para la verificación de enseñanzas oficiales en Catalunya. Una vez la enseñanza ha sido verificada, la Gen eralitat lo tien e que au torizar para que se pueda implantar, de acu erdo con los criterios de programación que establezca yla disponibilidad de financiación. Las enseñanzas autorizadas se inscriben en el Registro de Universidades, Centros y Titulaciones (RUCT) d el min isterio competen te en materia de un iversid ades, para poderser impartido. El objetivo de la verificación es garantizarquelas nuevas enseñanzas estén formulados de acuerdo con los requerimientos del EEES, el marco de calificaciones, y que haya una consistencia en tre los conten idos y los objetivos, de acuerdo con el planteamiento de las diferentes disciplinas.

Desde el inicio de este programa hasta el día de hoy, AQU Catalunya ha verificado 78 grados, 483 másteres y 195 doctorados.

- Seguimiento. El marco legal vigente establece que las agencias de evaluación de la calidad tien en que hacer un seguimien to de los títulos registrados, basándose en la información pública dispon ible, hasta el momento en que se tengan que someter a la evaluación para renovar la acreditación.

El Marco VSMA considera quela universidad es la primera re sponsable del proceso de seguimiento de sus titulaciones, mientras que AQU Catalunya actúa 
como agente externo degarantía de la calidad. Así, anualmente, los responsables de cada titulación elaboran un informe de seguimiento con el contenido siguiente: un resumen de la planificación de la titulación con la adición de todas las modificacion es in tro du cidas con posterioridad; los in dicad ores cuan titativos y el resto de información escrita (interna y/o pública) que refleje el desarrollo ordinario de las titulaciones; la valoración cualitativa de los indicadores y del resto de información que efectúen los agentes previstos en el SGIC de la un iversidad o, en el caso de una evaluación externa, una comisión de expertos; las propuestas de mejora que se deriven de la valoración que se hace del seguimiento de la titulación, incluyendo todas las propuestas de modificación.

De tod os los informes recibidos por parte de las universidades, AQU Catalunya analiza periódicamente y, si procede, realiza una evaluación in dividual en los casos siguientes: informes de las titulacion es oficiales que contengan propuestas de modificación sustancial; una muestra representativa de los otros informes de segu imien to de las titulaciones oficiales, prestan do una aten ción especial a las que presenten propuestas de modificación no sustancial; e informes de las titulaciones que se tengan que someter a evaluación externa (con visita) de su centro.

Adicionalmente, cada universidad elabora un informe que recoge una valora ción global del despliegue de las suyas. AQU Catalunya emite de forma periódi ca un informe de valoración de seguimiento para cada universidad, que puede proponer acciones de mejora para implementar a escala transversal.

- Modificación. Las universidades pueden hacer modificaciones a sus titulaciones. Si las modificaciones afectan aspectos como la definición administrativa o las características acad émicas esenciales de un título, las universidad es lo tienen que someter a un nuevo proceso de verificación. Para el resto de modificaciones, AQU Catalunya evalúa las propuestas de las universidades tomando como referencia la metodología desarrollada para la verificación. Si la evaluación de las modificaciones es favorable, las universidades pueden implantarlas directamente.

- Acreditación. AQU Catalunya es la responsable del proceso de acreditación de las titulaciones, que tien e que llevarse a cabo, en el caso de grados y doctorados, antes de los seis años a contar desde su verificación inicial (o después de acreditación) y, en el caso de máster, antes de los cuatro años. Para mantener la acreditación, los títulos tienen que obtener un informe positivo una vez se haya comprobado que el plan de estudios correspondiendo se está llevan do a cabo de acuerdo con su proyecto inicial, mediante una evaluación que tiene que incluir una visita externa a la institución.

Así, la Agencia organiza cada año visitas externas a centros docentes para eva asegu rar que todos los títu los universitarios han sido evaluados externamente, 
como mínimo una vez, dentro del periodo que marca la normativa legal. Estas visitas externas a las titulaciones se organizan por centros (facultad es y escue las) y las realiza un comité de evaluación externa que se constituye ad hoc para académico, estudiante y profesional - y un secretario.

En el 2016 se ha llevado a cabo la última revisión de la Guía para la acreditación de las titulaciones oficiales de grado y máster (AQU, 2016), con el fin de incor porar mejoras a partir de las deficiencias detectadas. Los estándares que se evalúan corresponden a seis dimensiones que están relacionadas con los procesos previos de verificación y segu imien to: calidad del programa formativo, pertinen cia de la información pública, eficacia del sistema de garantía interna de la calidad de la titulación, adecuación del profesorado al programa formati vo, eficacia de los sistemas de apoyoal ap rendizaje y calidad de los resultados del programa formativo.

Ad emás de estas dimensiones, se puede solicitar evaluar las titulaciones en tres dimensiones adicionales, cuyos criterios de evaluación se han aprobado: desa rrollo e inserción laboral, interacción entre investigación y docencia e interna cionalización.

El comité, al finalizar la visita, elabora un informe de visita externa valorando cada uno de los están dares evaluados. A partir de este informe, cada comisión específica por ámbito -Artes y Humanidades, Ciencias Sociales y Jurídicas, Ciencias, Ciencias de la Salud, Ingeniería y Arquitectura, y doctorad os - emite de forma colegiada el informe de acreditación, que puede ser favorable o des favorable y que se estructura en cuatro niveles: acreditado con excelencia, acreditado, acreditado con condiciones y no acreditado.

Para las titulaciones acreditadas con condiciones, sus respon sables y AQU Cata lunya acuerdan un plan de mejora que se tendrá que implantar en un plazo máximo de dos años. Una vez finalizado este periodo, las titulaciones tendrán que presentar un informe de seguimien to que evidencie los resultados de las acciones implantadas. Desde el inicio de este programa hasta el día de hoy, AQU Cata lunya ha acreditad o favorablemente 134 Grados y 122 másters y no ha acreditado un grado.

El gráfico siguiente muestra la evolución del número de títulos verificados, mo dificados y acreditados hasta el 2015. 


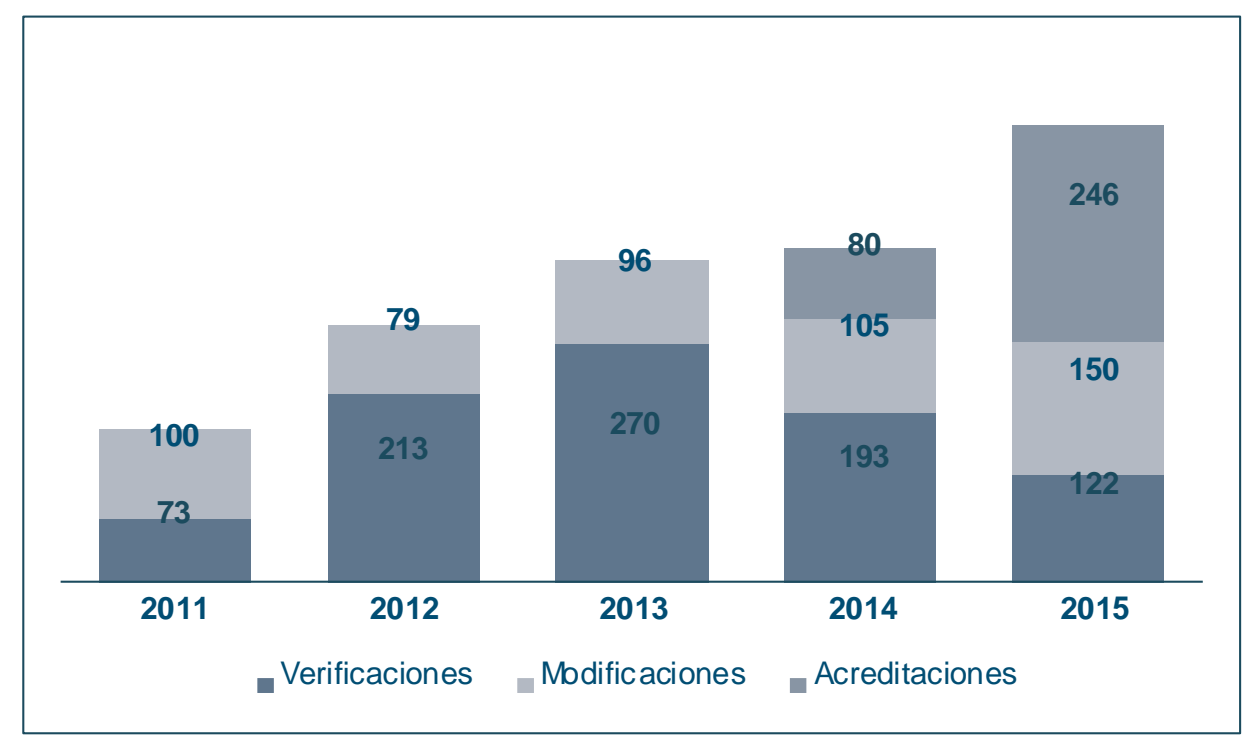

Por último, debe destacarse que, con el objetivo de dar publicidad y facilitar el acceso a los informes quela Agencia emite en el marco de los diferentes procesos de evaluación de las titulaciones, la Agencia dispone de un espacio específico en su web con un buscadorportipo de titulación, por tipo de programa y por universidad, que permite un acceso rápido y directo a todos los informes de evaluación elaborados por AQU Catalunya.

\subsection{Calidad del profesorado}

En este ámbito AQU Catalunya desarrolla fundamentalmente los procesos siguientes:

- Evaluación previa a los procesos de selección para la contratación de profeso rado en las universidades públicas y privadas catalanas (2003-actualitad). Este programa tiene como objetivo evaluar el currículum científico y acad émico de las personas que lo solicitan, para acreditar que han alcanzado el nivel de la categoría contractual a la cual optan:lector, agregad o y cated rático. Duran te el period o 2003-2007, AQU Catalunya evaluó el profesorado de las universidades privadas en los términos previstos en los convenios firmados con cada uno de estos centros y, a partir del 2008, la evaluación del profesorado de las universo dades privadas se ha integrado a los procesos de evaluación del profesorado. En el siguiente gráfico se muestra la evolución de estas evaluaciones desde el inicio del programa. 


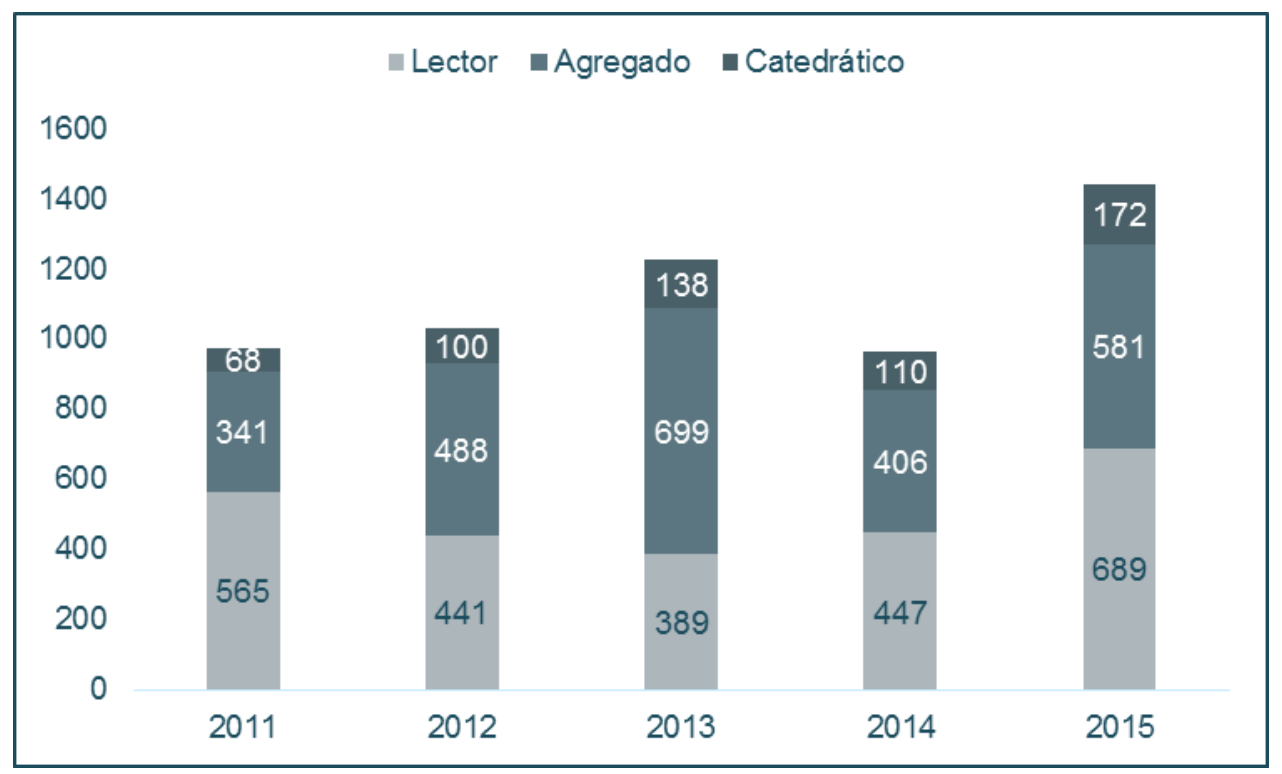

- Evaluación de los méritos individuales de docencia, investigación y gestión del personal docente e investigador, funcionario y contratado, de las universidades públicas catalanas en el marco de las retribuciones adicionales del profesorado (2006-actualitad). La Agencia evalúa directamente los méritos de investigación, notifica el resultado de la evaluación a las personas interesadas de acuerdo con el procedimiento establecido y comunica a cada universidad las evaluaciones favorables obtenidas por el profesorado respectivo, a fin de que el Consejo de Gobierno pueda formular la propuesta de asignación al Consejo Social. Con respecto a los méritos de docencia y gestión, la Agencia certifica la evaluación que hacen las universidades. En el caso de la docencia, las universidades públicas aplican los modelos que recogen los respectivos Manuales de evaluación docente, acreditados por AQU Catalunya.

\subsection{Generación de conocimiento}

AQU Catalunya elabora y promueve la elaboración de estudios de análisis del sistema universitario con el objetivo de dar información útil para la toma de decisiones de un iversidades y stakeholders. Los principales ámbitos en los cuales se trabaja son los siguientes:

- Encuestas de inserción laboral. Desde el año 2001 AQU Catalunya y las universidades catalanas realizan trienalmente $(2005,2008,2011$ y 2014$)$ la encuesta conjunta de inserción laboral a sus graduad os y graduadas, para conocer, entre otras cuestiones, el empleo la calidad de dicho empleo y su grado de satisfacción con respecto a la formación recibida. 
Por ejemplo, en la última encuesta realizada durante el primer trimestre del 2014, participaron los graduados de los antiguos ciclos que finalizaron los estu dios en el año 2009 (excepto los de Medicina, que son del año 2007) y los doc tores que acabaron de estudiar en los años 2009 y 2010. Asimismo, y como novedad de esta edición, también se encuestaron a los graduados de máster que finalizaron los estudios en el 2010 y en el 2011.

En total, en esta quinta edición se analizaron las respuestas de 17.295 gradua dos de una población de referencia de 31.734 (55\% de respuesta y error mues tral del 0,51\%) y de 2.107 doctores de una población de 3.104 (69\% de respues ta y error muestral del 1,5\%).

Además de las convocatorias que AQU Catalunya lanza para perfeccionar el conocimien to y el diagnóstico de la inserción laboral de los graduados universo tarios, también pone a disposición de la comunidad académica e investigadora de las universidades catalanas toda la información, con la in tención de fomen tar su uso y explotación.

- Encuestas a los empleadores. La Obra Social "la Caixa", por medio del convenio que tien e con la Generalitat de Catalunya, ha fin an ciado AQU Catalunya para llevar a cabo un estu dio sobre la inserción laboral de los graduad os universitarios desd ela perspectiva de los empleadores. Este estu dio, que complementa la encuesta trienal de inserción laboral a las personas graduadas de las universidad es catalanas, preten de dar información a las un iversidades sobre cuál es la percepción del mercado laboral respecto de las competencias y la formación universitaria de los titulados. La finalidad es que las universidades puedan desarrollar políticas de mejora en el sistema universitario con respecto a la oferta académica y a los programas formativos, y acercar así la formación universitaria y el mercado laboral. Las universidades catalanas y el Gobierno de la Generalitat de Catalunya (Departamento de Economía y Conocimiento, Departamento de Empresa y Empleo, Departamento de Enseñanza, Departamentode Función Pública y Departamento de Salud) se han implicad o fu ertemente para impulsar este proyecto, que tiene que permitir también la comparación de los datos del sistema universitario catalán en el ámbito europeo, dado que la encuesta recoge las dimensiones principales del estudio "Eurobarómetro de la Comisión Europea (The Gallup Organization, 2010) titulado “Employers' perception of graduate employability" que se hizo en el año 2010 a los 27 estados de la Unión Europea.

- Encuesta de satisfacción de los estudiantes. AQU Catalunya inició en el año 2012, jun to con las universidadescatalanas, la definición deun instrumento $p$ ara conocer el grado de satisfacción de los estudiantes después de su graduación. El objetivo principal del proyecto era disponer de una encuesta que permitiera la recogida de indicadores comunes sobre la satisfacción de la pobla- 
ción graduada y facilitar, tanto al sistema universitario catalán como a los responsables de las titulaciones, in formación para la mejora de las enseñanzas. El instrumento fue consensuad o por el conjunto de universidades catalanas, públicas y privadas, por medio del grupo de trabajo creado por AQU Catalunya con esta finalidad, y fue aprobado por AQU Catalunya.

El cuestionario tien e un total de 22 ítems que inclu yen cuestiones relacionadas con cuatro bloques o dimensiones: el desarrollo operativo dela titulación (plan de estudios, coordinación, volumen de trabajo exigido, metodología docente, evaluación de los apren dizajes y tutoría), la consecución de competencias, los servicios y equipamientos, y la satisfacción global.

A la vista de la previsión de encuestas de satisfacción que necesita el sistema universitario catalán, se ha constituido el Comité Técnico de Encuestas, que tien e como objetivo mejorar la coordinación en los planteamientos, recogida y análisis de los datos de las diferentes encuestas que se desarrollen y la o ptimi zación de los recursos técnicos de las universidades y de la Agencia.

- WINDDAT, indicadores docentes para el desarrollo y el análisis de las titulaciones. En el año 2012 AQU Catalunya puso en funcionamiento el WINDDAT, una web pública en que se muestran datos sobre demanda, nota de corte, plazas ofrecidas, tasa de rendimiento, etc., a partir de las cuales los responsables universitarios pued en elaborar sus informes de seguimiento, y posteriormen te los de acreditación, y comparar los datos de su enseñanza con el resto de los que se ofrecen en Catalunya.

- Estudios. AQU Catalunya ha impulsado la realización de estudios sobre el sistema universitario catalán en ámbitos específicos para obtener una imagen más objetiva del sistema. Concretamente, se han realizado estu dios sobre rendimien to acad émico, el abandono del estudiantado y el punto de vista del estu dian tado. También ha realizado análisis del sistema un iversitario a partir de la perspectiva de la evaluación y sobre los procesos de evaluación del profesorado universitario. Uno de los últimos estudios en este ámbito ha sido el análisis de las trayectorias cu rriculares del profesorado universitario a partir de los currículums que se han presentado a los programas de evaluación del profesorado de AQU Catalunya.

- Materiales metodológicos. AQU Catalunya diseña los materiales metodológicos con los cuales realiza los procesos de evaluación de la calidad en Catalunya. Hasta el momento, ha desarrollado más de una trenta de guías de evaluación de la calidad, y también instrumentos para dar apoyo a las universidades catalanas a la hora de orientar la evaluación de ámbitos específicos del sistema, como los marcos generales, las guías para el diseño de programas de formación y las guías de evaluación de las competen cias. 
- Talleres y jornadas. Aparte de jornadas específicas, AQU Catalunya organiza cada año un taller de reflexión y debate con las universidades catalanas para propagar el conocimiento y trabajar de manera coordinada con las personas encargad as de llevar a cabo los diferentes procesos de mejora de la calidad en las universidades.

Todos los materiales que AQU Catalunya ha elaborado en el marco de la Gestión del conocimiento están disponibles en la web.

\subsection{Internacionalización}

Con el fin de mantenersus procesos de aseguramiento de la calidad alinead os con las mejores prácticas eu ropeas, AQU Catalunya participa en proyectos internacionales. Al mismo tiempo, esta presencia internacional se hace con la voluntad de contribuir al desarrollo de nu evos esqu emas para el EEES. Así, AQU Catalunya desarrolla su dimensión internacional asumiendo diferentes tipos de actividades que se agrupan en las categorías siguientes:

- Relaciones institucionales. AQU Catalunya coopera, principalmente, en el ámbito europeo y con agencias o entidades con objetivos comunes con el fin de desarrollar proyectos relevantes y que ap orten valor añadid o al sistema universitario catalán. AQU Catalunya es miembro de pleno derecho de ENQA desde su fundación el año 2000, y cuenta con un representante en el Consejo de Gobierno de EQAR el registro eu ropeo de agencias de calidad. La INQAAHE es una asociación internacional con más de 200 organizaciones que trabajan en el aseguramiento de la calidad de la educación superior, la mayor parte agencias. AQU Catalunya es miembro d esde el año 1998, y ocupa el Secretariado desd e el 2013. AQU Catalunya se in tegró en el año 2010 en ECA, que tiene como objet ivo el reconocimiento mutuo de las decisiones de acreditación y de aseguramientode la calidad.ECA desarrolla sus actividades mediante grupos de trabajo. Cada uno de estos grupos está formado por representantes de los organismos miembros del consorcio.

- Proyectos internacionales: AQU Catalunya coopera con otros organ ismos, a petición de estos o a petición propia, en la realización de proyectos, con el objetivo de que aporten valor añadido a las activid ades desarrolladas por la Agencia o bien que den apoyo a países que estén en fase de desarrollo de estas actividades. La participación en proyectos internacionales se estructura en cuatro ámbitos deactuación: la in ternacionalización (in tegración del asegu ramien tode la calidad en los procesos de internacionalización de las universidades), la cooperación (difusión yextensión in ternacional del modelo catalán y eu ropeo de asegu ramien tod ela calid ad fuera de la Unión Europea), la in novación (de los procesos de asegu ramien tode la calidad con colaboración con otras agencias) y el bench marking (identificación de referentes internacionales para el aseguramientode la calidad en Catalunya). 
- Evaluaciones y certificaciones internacionales. La Agencia evalúa de forma periódica programas fuera del Sistema Universitario Catalán. Al mismo tiempo mantiene diferentes tip os de colaboraciones con agencias, asociacion es $u$ otro tipo de instituciones con el fin de facilitar a las universidades catalan as la obtención de reconocimientos internacionales (labels, certificaciones...).

\section{Conclusiones}

De la legislación estatal destacan sobre todo dos ámbitos que inciden en la actividad de AQU Catalunya, que son los relacionados con la evaluación de titulaciones y la evaluación del profesorado universitario.

Con respecto a la evaluación de las titulaciones universitarias oficiales, el Gobierno estatal ha establecido que las titulaciones universitarias tien en que seguir un sistema de verificación, modificación, seguimiento y acreditación. Para desarrollar este mandato legal de acuerdo con los "European Standards and Guidelines for the external Quality Assurance of Higher Education" (ESG), como se ha comentado, AQU Catalunya ha diseñado en colaboración con las universidades y el Gobierno de la Generalitat de Catalunya el Marco para la verificación, el seguimiento, la modificación y la acreditación de las titulaciones (Marco VSMA), que ha sid o aprobad o en el 2010 por el Consejo de Dirección de la Agencia y que se está desplegando desde 2011.

Por otra parte y a diferencia de la mayoría de los países europeos, en España las agencias de calidad evalúan al profesorad o universitario. Desdeel 2003 AQU realiza evaluaciones previas a los procesos de selección de profesorad o contratado de las universidades públicas catalan as para las figu ras de colaborador, lector, agregad o y cated rático; evalúa el profesorado de las universidades privad as para dar cumplimien toa la LUC, y desde el año 2006 evalúa los méritos individuales de docencia, investigación y gestión del personal docente e investigad or, funcionario y contratado, de las universidades catalanas en el marco de las retribuciones adicionales del profesora do.

Ahora bien, como se destaca en este artículo, estas no son las únicas dos funciones de AQU Catalunya, sino que su objetivo es ir mucho más allá, con el objetivo de dar herramientas y sop ortea la mejora del Sistema Universitario Catalán. Se han desta cado en el artículo aspectos de gran impacto como la encuesta de in serción laboral, o la encuesta más reciente hecha a las organizaciones que dan empleo a los graduados universitarios. Ahora bien, todavía quedan muchos más retos por alcanzar, como por ejemplo la evaluación de la investigación de los departamentos universitarios, de la cual AQU Catalunya ya ha llevad o a cabo una prueba piloto en todos los departamentos de una universidad del Sistema Universitario Catalán o el portal EUC (Estudios Un iversitarios de Catalunya) para la difusión a la socied ad de la calidad de los programas que se imparten en Catalunya, evidenciando una vez más que, con la legislación a ctual, es posible profund izaren muchos aspectos para con segu ir la mejora de la calidad universitaria. 


\section{Bibliografía}

AQU (2010), Marco para la verificación, el seguimiento, la modificación y la acreditación de los títulos oficiales

AQU (2015), Plan Estratégico de AQU Catalunya 2015-2018

AQU (2016), Guia para la acreditación de las titulaciones universitarias oficiales de grado y máster

Ley Orgánica 6/2001, de 21 de diciembre, de universidades.

Ley $1 / 2003$, de 19 de febrero, de universidades de Catalunya.

Ley 15/2015, del 21 de julio, de la Agencia para la Calidad del Sistema Universitario de Catalunya.

The Gallup Organ ization (2010). Employers' perception of graduate employability. Analytical report. Eu ropean Commissio. 